\title{
An efficient closure based method for inverse climate modelling
}

\author{
$\underline{\text { Meelis J. Zidikheri }}^{\text {a }}{ }^{\text {, Jorgen S. Frederiksen }}{ }^{\mathrm{b}}$ \\ ${ }^{a}$ Centre for Australian Weather and Climate Research, Australian Government Bureau of Meteorology, GPO \\ Box 1289, Melbourne, Victoria 3001, Australia \\ ${ }^{\mathrm{b}}$ Centre for Australian Weather and Climate Research, CSIRO Marine and Atmospheric Research, PMB 1, \\ Aspendale, Victoria 3195, Australia \\ Email: m.zidikheri@bom.gov.au
}

\begin{abstract}
In this study, a new method is developed for attributing changes in climate states by calculating the anomalous forcing functions responsible for these changes. The method relies on an iterative procedure to calculate improved estimates of the forcing function starting from an initial estimate. Running a sequence of climate models can be computationally demanding and therefore computational efficiency is an important ingredient in formulating a tractable inverse modelling scheme. It is therefore crucial to initialise the iterative procedure with a forcing function that is a good estimate of the correct forcing function to reduce the number of iterations required. This is achieved by formulating a statistical closure scheme. A statistical closure scheme enables one to overcome the closure problem in which statistical moments of higher order appear in the prognostic equations for the statistical moments of a given order. For example, the equation for the mean field depends on second-order moments and the second-order moment equations in turn depend on third-order moments. The closure scheme that we employ involves a linearisation of the second-order moment terms, which physically represent the feedback of transient eddies on the mean circulation, in terms of the mean fields. The parameters required in this closure scheme are calculated by employing perturbation experiments. We demonstrate that the closure scheme leads to simulated climate states that are in close agreement to idealised and observed climate states with pattern correlations generally greater than 0.8 . These closure based estimates may then be further improved by employing the iterative method.
\end{abstract}

Keywords: Inverse modelling, climate change, attribution, closure, eddy parameterisation 


\section{INTRODUCTION}

Attributing the causes of observed climate change to a variety of physical and dynamical processes is a problem of great contemporary significance in climate science. As a case in point, in the southern hemisphere there have been dramatic changes in the circulation and rainfall during the second half of the 20th century, particularly in the winter and autumn (Frederiksen and Frederiksen, 2007). Understanding the extent to which these changes are due to internal variability and the extent to which a systematic shift in the climate and rainfall is occurring due to increasing anthropogenic greenhouse gases is of major scientific and policy concern.

Current approaches to climate change attribution have formally relied on the statistical methods described by Hasselman (1993), Hegerl et al. (1996), and Hasselman (1997). In these approaches, the climate change signal for a particular climate change driver must be identified in the climate change data. Recently, new methods for attacking the attribution problem have been proposed (Zidikheri and Frederiksen, 2011, 2013a,b). In this approach, forcing functions associated with the climate change drivers are calculated from climate change data using inverse modelling techniques. It has been proposed that these forcing functions can then be used to identify the climate change drivers.

The inverse modelling approach of Zidikheri and Frederiksen can be accomplished in two ways. In the simplest version, the forcing function for a particular variable is calculated iteratively; that is, it is adjusted at each iteration step by introducing a term proportional to the difference between the observed and simulated climatic value of the variable. This method has been shown to work extremely well but it requires a good initial estimate of the forcing function for rapid convergence.

The second inverse modelling approach relies on finding a suitable statistical closure scheme for the equations of motion. The dynamical equations describing the climate, and indeed all high Reynolds number turbulent flows, are in general statistically unclosed (McComb, 1990). However, if a closure scheme can be found then the climatic forcing function can be directly calculated from the climate state. The problem of relating the forcing function to the climate state has in the past been approached by invoking the fluctuation-dissipation theorem as proposed by Leith (1975). This approach has been implemented in various contexts with some success (Bell, 1980; Gritsoun, 2001; Gritsun and Branstator, 2007; Gritsun et al., 2008) although it has been noted that in some geographical locations serious discrepancies occur. A relatively new approach to the problem is provided by statistical dynamical closure models, in particular the Quasi-diagonal Direct Interaction Approximation (QDIA) of Frederiksen (1999) and Frederiksen (2012a), which may be derived from the more complex Self Energy (SE) closure model of Frederiksen (2012b).

These closure models provide useful insight into the correct form of the closure scheme. However in the studies of Zidikheri and Frederiksen $(2011,2013 a$ ab) and in this study, rather than deriving closed expressions for the coefficients in the closure scheme, the coefficients are instead calculated by sampling suitable perturbation experiments and linear regression of the relevant quantities obtained from the samples. In this study we compare and contrast the spectral approach of Zidikheri and Frederiksen $(2011,2013 \mathrm{~b})$ with the grid-point approach of Zidikheri and Frederiksen (2013a). We implement the methodologies in the context of the July 1959-1968 and 1975-1984 climate states using a two-level quasigeostrophic potential vorticity model. This study differs from the earlier study of Zidikheri and Frederiksen (2011) — which considered similar climatic periods, in its implementation of the observed climatic potential vorticity which is herein treated in a more realistic manner. It also differs in its treatment of the closure scheme which is considerably improved in its generality, taking advantage of the advances described in Zidikheri and Frederiksen (2013a,b).

The plan of this paper is as follows. In Section 2, we described the two-level quasigeostrophic potential vorticity model and relax the model towards the July 1959-1968 and 1975-1984 climate states using the iterative method. In Section 3 we describe the spectral and grid-point closure methods. In Section 4 we present the results obtained with these closure methods. Section 5 presents the conclusions.

\section{THE TWO-LEVEL QUASIGEOSTROPHIC MODEL}

\subsection{General model description}

We employ the two-level quasigeostrophic potential vorticity equations as discussed in detail by Salmon (1998). For flow on the spherical earth, these equations take the form

$$
\frac{\partial q^{j}}{\partial t}=-J\left(\psi^{j}, q^{j}+h^{j}\right)-2 \frac{\partial \psi^{j}}{\partial \lambda}-\left[\alpha^{j}+(-1)^{\rho} \nu_{0}^{j} \nabla^{2 \rho}\right] \zeta^{j}+\kappa\left(\tilde{q}^{j}-q^{j}\right),
$$


M.J. Zidikheri and J.S. Frederisen, An efficient closure based method...

where $j=1$ is the upper level and $j=2$ is the lower level. Here, the quasigeostrophic potential vorticity at level $j$ is defined as

$$
q^{j}=\nabla^{2} \psi^{j}+(-1)^{j} F_{c}\left(\psi^{1}-\psi^{2}\right),
$$

where $\psi^{j}$ are level-dependent streamfunctions, $\zeta^{j}=\nabla^{2} \psi^{j}$ are relative vorticities, and $F_{c}$ is a constant factor. This factor controls the growth rate of baroclinic instabilities and is inversely proportional to the midlatitude static stability.

The Jacobian operator is defined as $J(X, Y)=\frac{\partial X}{\partial \lambda} \frac{\partial Y}{\partial \mu}-\frac{\partial Y}{\partial \lambda} \frac{\partial X}{\partial \mu}$, where $\lambda$ is the longitude, $\mu=\sin \phi$, with $\phi$ being the latitude; $X$ and $Y$ are general fields. The model includes drag specified by $\alpha^{j}$ and prescribed viscosities $\nu_{0}^{j} ; \rho$ is a positive integer that describes the order of the Laplacian operator. The topographic contribution to the vorticity is denoted by $h^{j}$ and is given by $h^{j}=2 \mu H^{j} / H_{0}$, where $H^{j}$ is the topographic height and $H_{0}$ is a scale height.

We relax the potential vorticity towards the time-independent field,

$$
\tilde{q}^{j}=\nabla^{2} \tilde{\psi}^{j}+(-1)^{j} F_{v}\left(\tilde{\psi}^{1}-\tilde{\psi}^{2}\right)
$$

on a time scale, $\kappa^{-1}$. This field is referred to as the forcing function and is a parameterisation of the effects of heating, and takes into account the effect of varying static stability through the spatially variable instability factor, $F_{v}$. This is to ensure that the flow is relaxed as closely as possible towards the correct potential vorticity values everywhere on the globe.

The model is integrated in the spectral domain, and the spectral model is obtained by expanding the fields $\psi^{j}$, $\zeta^{j}, q^{j}$, and $h^{j}$ in terms of spherical harmonics, yielding the discrete spectral fields $\psi_{m n}^{j}, \zeta_{m n}^{j}, q_{m n}^{j}$, and $h_{m n}^{j}$, where $m$ is the longitudinal wavenumber and $n$ is the total wavenumber.

\subsection{Useful diagnostics}

It is useful to calculate the barotropic (+) and baroclinic (-) streamfunctions, which in spectral space are given by

$$
\psi_{m n}^{ \pm}=\frac{\psi_{m n}^{1} \pm \psi_{m n}^{2}}{2}=-\frac{q_{m n}^{1} \pm q_{m n}^{2}}{2 c_{m n}^{ \pm} n(n+1)},
$$

where $c_{m n}^{+}=1$ and $c_{m n}^{-}=1+\frac{2 F_{c}}{n(n+1)}$. From, the baroclinic streamfunction, the quasigeostrophic temperature may be calculated as

$$
T=\frac{2 k \Omega \mu \psi^{-}}{R}+T_{0}
$$

This relation follows from imposing the quasigeostrophic approximation to the hydrostatic part of the atmospheric primitive equations (Salmon, 1998). Here $R$ is the thermodynamic gas constant, $\Omega$ is the angular velocity of the Earth, and $k=2.5$ whose value follows from considering the difference between the pressure levels at $j=1$ and $j=2$. The global temperature, $T_{0}$, cannot be uniquely determined from the quasigeostrophic model; however, the spatial temperature anomaly $T-T_{0}$ is uniquely determined and it is in this sense that we shall refer to the temperature in the remainder of this paper. It is also useful to calculate the longitudinal and latitudinal winds, respectively, as

$$
u^{ \pm}=-\frac{1}{a} \frac{\partial \psi^{ \pm}}{\partial \phi} \quad \text { and } \quad v^{ \pm}=\frac{1}{a} \frac{\partial \psi^{ \pm}}{\partial \lambda} .
$$

\subsection{Inverse modelling by iteration}

The quasigeostrophic model can be run as an inverse model by treating the forcing function, $\tilde{q}^{j}$, as an unknown variable and adjusting its value at each point so that the simulated climatological value of the potential vorticity, $\left\langle q^{j}\right\rangle$, approaches a specified value $\overline{q^{j}}$. At the $(i+1)$-th iteration step,

$$
\tilde{q}^{j}(i+1)=\tilde{q}^{j}(i)+\kappa\left(\overline{q^{j}}-\left\langle q^{j}(i)\right\rangle\right)
$$

The 0 -th iterate, $\tilde{q}_{m n}^{j}(0)$, can be chosen arbitrarily, but clearly the scheme will converge faster the closer it is to the 'correct' value for which the term in the brackets in Eq. (7) vanishes. 
(a)

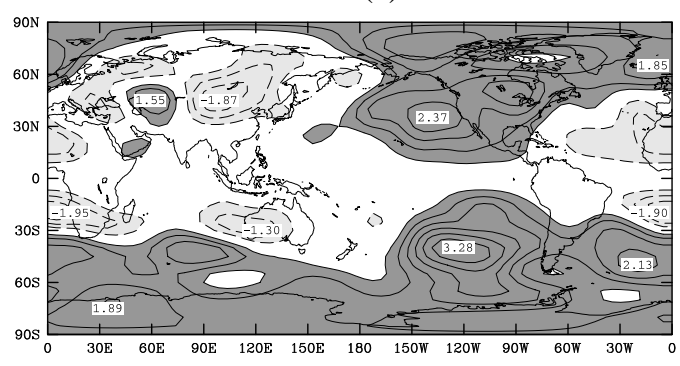

(b)

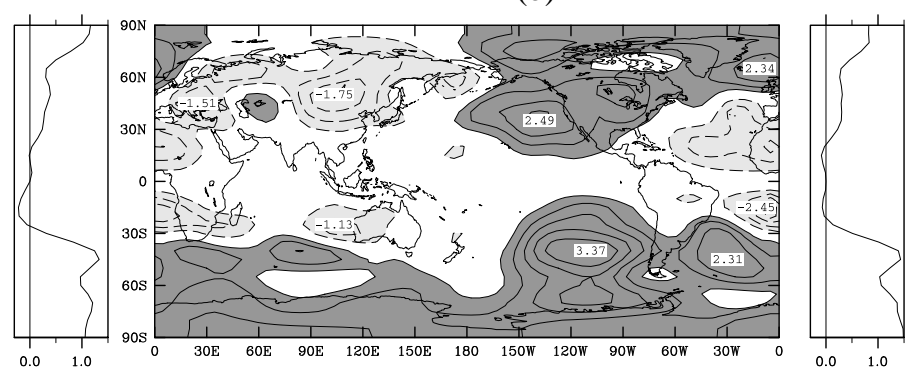

Figure 1. July 1975-1984 - 1959-1968 anomalous climatological temperature (in K) as obtained from (a) reanalysed data and (b) quasigeostrophic model.

\subsection{Results for July 1959-1968 and 1975-1984 climate states}

We focus on the decadal periods 1959-1968 and 1975-1984 for the month of July, for which some of the greatest southern hemisphere circulation changes have been observed (Frederiksen and Frederiksen, 2007). Using NCEP/NCAR reanalysis data for these periods, we calculate the 'observed' potential vorticity, $\overline{q^{j}}$, using the variable instability factor, $F_{v}$, which is chosen for simplicity to be longitudinally symmetric. Its form in the latitudinal direction is chosen so as to reproduce the observed climatic variability characteristics as closely as possible. Equation (7) is then used to calculate the forcing function $\tilde{q}^{j}$ by iteration. We set the initial iterate to be equal to the observed climatic value $\overline{q^{j}}$. To speed up the iteration, we initially use a simulation sampling time of 200 days, but progressively increase the sampling time to 2000 days in the final few iteration steps. The numerical values of the various model parameters are as listed by Zidikheri and Frederiksen (2011). Figure 1 shows the results displayed as the climatological July 1975-1984 - 1959-1958 temperature anomaly for both the reanalysis and simulated data. The simulated and observed climate change patterns shown in Fig. 1 are in good agreement indicating that the inverse modelling approach has been successful. The anomalous temperature patterns indicate a high-latitude warming response, and this more pronounced - and more longitudinally symmetric - in the southern hemisphere. The corresponding forcing function for this case is shown in Fig. 2(a); its form is discussed in Section 4.

\section{CLOSURE BASED INVERSE METHOD}

\subsection{Introduction}

It can be shown that by computing the average of Eq. (1) at statistical steady state, and writing all the timedependent fields as the sum of an average mean term (denoted by angular brackets) and a transient term (denoted by a hat) we obtain the following expression for the anomalous forcing function, defined as the difference between the forcing function of a climate state relative to some base state:

$$
\delta \tilde{q}^{j}=\delta T^{j}+\delta J\left(\left\langle\psi^{j}\right\rangle,\left\langle q^{j}\right\rangle+h^{j}\right)+B \frac{\partial \delta\left\langle\psi^{j}\right\rangle}{\partial \lambda}+\left[\alpha^{j}+(-1)^{\rho} \nu_{0}^{j} \nabla^{2 \rho}\right] \delta\left\langle\zeta^{j}\right\rangle+\kappa \delta\left\langle q^{j}\right\rangle,
$$

where $T^{j}=\left\langle J\left(\hat{\psi}^{j}, \hat{q}^{j}\right)\right\rangle$ is the feedback of the transients on the mean field and the $\delta X=X-X_{0}$, with $X$ being a climatic state variable and $X_{0}$ being the corresponding variable for the base climate. Equation (8) is exact but is unclosed in the mean field due to the transient feedback term $T^{j}$ which depends on the second-order moments of $q^{j}$. If the forcing function is to be directly calculated from the mean field, then approximations to $T^{j}$ that depend on the mean field $\left\langle q^{j}\right\rangle$ need to be found. As mentioned in the introduction, such approximations have been developed by Zidikheri and Frederiksen $(2013 a, b)$ as described further below.

\subsection{Diagonal spectral closure}

This closure scheme was discussed in detail by Zidikheri and Frederiksen (2011, 2013b). It is formulated as a diagonal closure in spectral space following the QDIA closure model as follows:

$$
\delta\left\langle T_{m n}^{j}\right\rangle=-\sum_{k=1}^{2} D_{t}^{j k}(m, n) \delta\left\langle q_{m n}^{k}\right\rangle+f_{t}^{j}(m, n) .
$$


Here, the indices $j, k=1,2$ and are understood to run over the barotropic and baroclinic components; that is, $j=1$ (and $k=1$ ) implies the barotropic field and $j=2$ (and $k=2$ ) implies the baroclinic field. The coefficients $D_{t}^{j k}(m, n)$ and $f_{t}^{j}(m, n)$ do not have closed expressions as in the QDIA but are determined by linear regression of an ensemble of perturbation experiments as described further below.

\subsection{Quasi-diagonal grid-point closure}

This closure scheme was discussed in detail by Zidikheri and Frederiksen (2013a). It is formulated in terms of physical grid points, denoted by $\mathbf{r}$, as follows:

$$
\delta\left\langle T_{x}^{j}(\mathbf{r})\right\rangle=\sum_{k=1}^{2} A^{j k}(\mathbf{r}) \delta\left\langle x^{k}(\mathbf{r})\right\rangle+\sum_{k=1}^{2} B^{j k}(\mathbf{r}) \delta\left\langle\bar{x}^{k}(\mathbf{r})\right\rangle+b^{j}(\mathbf{r}),
$$

where $A^{j k}(\mathbf{r}), B^{j k}(\mathbf{r})$, and $b^{j}(\mathbf{r})$ are determined by linear regression as in the spectral method. The subscript $x$ in Eq. (10) implies that $T$ is calculated in the same 'space' as the variable $x$ on the right-hand side of Eq. (10). In Zidikheri and Frederiksen (2013a) it was shown that the best space to effect this parameterisation is the velocity space. Hence, we must perform the sequence of transformations outlined in Section 2.2 to transform $T$ from potential vorticity space into velocity space. In Equation (10) we have defined a new coarse-grained field $\bar{x}^{k}(\mathbf{r})=\sum_{\boldsymbol{r}^{\prime} \in \mathcal{R}} x^{k}\left(\boldsymbol{r}^{\prime}\right)$, where $\mathcal{R}$ is a region defined by the set of points $\left(\lambda^{\prime}, \phi^{\prime}\right)=\boldsymbol{r}^{\prime},(\lambda, \phi)=\boldsymbol{r}$ such that $\left|\lambda-\lambda^{\prime}\right| \leq \lambda_{m},\left|\phi-\phi^{\prime}\right| \leq \phi_{m}$, and $\lambda_{m}, \phi_{m}$ are values chosen so as to minimize the least-squares linear regression error. It was found empirically that values of $\lambda_{m}=\phi_{m}=45^{\circ}$ give optimal results. Note that a diagonal closure scheme analogous to the spectral one in Eq. (9) was also formulated in terms of grid points in Zidikheri and Frederiksen (2013a). However it was shown in that paper that the quasi-diagonal formulation of Eq. (10) gives superior results and hence the diagonal grid-point formulation will not be considered further in this paper.

\subsection{Sampling ensemble generation}

In this study we employ randomly perturbed simulations to generate an ensemble of samples from which the coefficients in Eqs. (9) and (10) may be evaluated by linear regression. We perturb the base climate forcing function, which we take to be the July 1959-1968 climate state forcing function, as follows in spectral space:

$$
\delta \tilde{q}_{m n}^{j}(i)=\Lambda_{i} \Gamma_{i} \exp \left(i \Theta_{i}\right)
$$

where $\Lambda_{i}=0.1$ ceiling $\left(\frac{i}{20}\right), \Gamma_{i}=\left|\tilde{q}_{m n}^{j}(0)\right| r_{i}$, and $\Theta_{i}=r_{i} \arctan \frac{\Im \tilde{q}_{m n}^{j}(0)}{\Re \tilde{q}_{m n}^{j}(0)}$. Here, $i$ is an integer between 1 and $N$, the number of samples; the ceiling function gives the next nearest integer value greater than its argument; $\tilde{q}_{m n}^{j}(0)$ is the complex spectral potential vorticity amplitude of the reference climate forcing function; and $r_{i}$ is a random number between -1 and 1 . The algorithm described by Eq. (11) for $N=100$ results in 100 samples in five batches of 20 samples each. Each batch consists of the reference climate forcing function scaled by a factor of between 0.1 and 0.5 (inclusive) and with randomly scrambled amplitude and phase.

\section{RESULTS AND GENERAL DISCUSSION}

The results are summarised in Table 1. We have calculated the pattern correlations between the anomalous forcing function as obtained by iteration and the corresponding forcing function as obtained with both the spectral and grid-point closure methods. The forcing functions are well reproduced by both closure methods, with pattern correlations of over 0.8 in all cases. We have also calculated the pattern correlations between the transient feedback term as obtained iteratively and as obtained using the closures. In both methods, we find that the barotropic feedback term is poorly reproduced by the closures. The baroclinic term is however relatively well reproduced, with the grid-point method outperforming the spectral method. It should be remembered that the goal of carrying out the closure calculations is to find a good first approximation for the forcing function that can then be further improved by the iterative method. In this case, both closure methods fullfil this function extremely well. However, in general there might be situations where the forcing function is more sensitive to the feedback term, in which case the grid-point method would be preferable over the spectral method as it would provide a better approximation to the forcing function, at least for the baroclinic term. The baroclinic term is of utmost importance because from it we can calculate the temperature forcing funcion, which is crucial for attribution studies. This representation of the forcing function is shown in Fig. 2 for the iterative method and the two closure methods. Both closure methods are seen to reproduce the overall 
M.J. Zidikheri and J.S. Frederisen, An efficient closure based method...

\begin{tabular}{ccccc}
\hline & Barotropic $(\mathrm{S})$ & Baroclinic $(\mathrm{S})$ & Barotropic $(\mathrm{G})$ & Baroclinic $(\mathrm{G})$ \\
\hline Feedback term & 0.21 & 0.52 & 0.22 & 0.65 \\
Forcing function & 0.85 & 0.83 & 0.87 & 0.87 \\
\hline
\end{tabular}

Table 1. Pattern correlations of anomalous transient feedback term and forcing function as obtained with spectral closure method (S) and grid-point closure method (G)

(a)

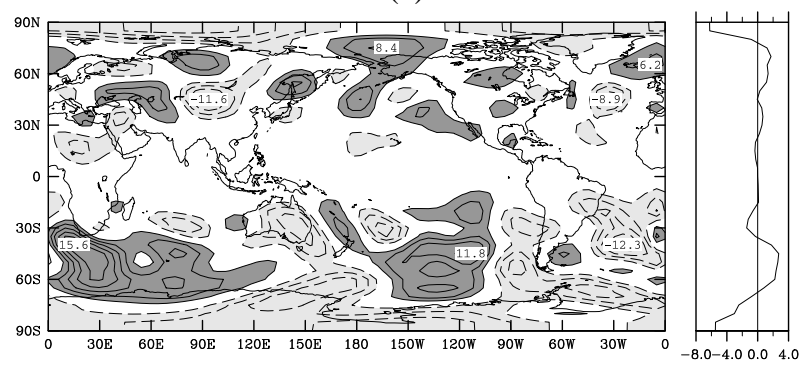

(b)

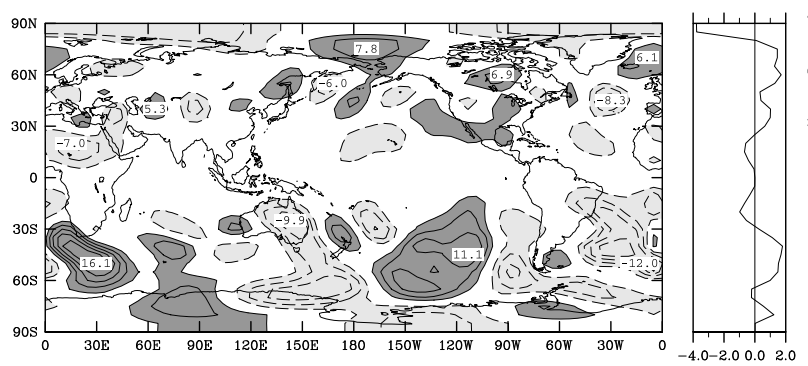

(c)

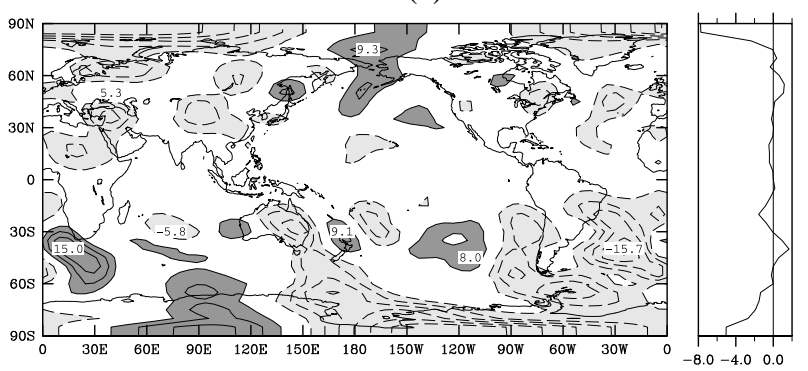

Figure 2. July 1975-1984 - 1959-1968 anomalous climatological effective temperature forcing functions (in $\mathrm{K}$ ) as obtained from (a) iterative method, (b) spectral closure method, and (c) grid-point closure method.

structure of the forcing function as obtained with the iterative method extremely well. The patterns shows a significant tendency towards longitudinally-symmetric high-latitude cooling over both hemispheres and some warming, albeit longitudinally asymmetric, over the midlatitudes. The midlatitude temperature gradient forcing appears to favour the more stable enhanced poleward warming configuration which would be consistent with a greenhouse warming signal. The high-latitude temperature forcing gradient is however in the opposite direction favouring the unstable enhanced poleward cooling configuration. It is difficult to conclude to what extent this polar cooling tendency is a result of a physical cooling tendency or a result of the inadequacy of the quasigeostrophic model at high latitudes. To explore these issues further we hope to implement the method in a primitive equation model in the future.

\section{CONCLUSION}

We have demonstrated how the forcing function, representing climate drivers in a climate model, may be obtained from climate change data by inverse modelling of the dynamical equations of motion. Firstly, this was successfully carried out using an iterative procedure. Secondly, we demonstrated how a good estimate of the forcing function may be obtained using various closure approximations to the first-order statistical moments of the dynamical terms. Pattern correlations of over 0.8 relative to the iteratively-calculated forcing function were obtained with these methods. These closure-based approximations would provide better initial estimates for the iterative method and thus significantly improve the computational efficiency of the inverse modelling procedure. 
M.J. Zidikheri and J.S. Frederisen, An efficient closure based method...

\section{ACKNOWLEDGEMENT}

The authors acknowledge support by the West Australian Department of Environment and Conservation under the IOCI-3 program and the ACCSP program of the Australian Department of Climate Change and Energy Efficiency.

\section{REFERENCES}

Bell, T. L. (1980). Climate sensitivity from fluctuation dissipation: Some simple model tests. Journal of the Atmospheric Sciences 37(8), 1700-1707.

Frederiksen, J. S. (1999). Subgrid-scale parameterizations of eddy-topographic force, eddy viscosity, and stochastic backscatter for flow over topography. Journal of the atmospheric sciences 56(11), 1481-1494.

Frederiksen, J. S. (2012a). Statistical dynamical closures and subgrid modeling for inhomogeneous qg and 3d turbulence. Entropy 14(1), 32-57.

Frederiksen, J. S. (2012b). Self-energy closure for inhomogeneous turbulent flows and subgrid modeling. Entropy 14(4), 769-799.

Frederiksen, J. S. and C. S. Frederiksen (2007). Interdecadal changes in southern hemisphere winter storm track modes. Tellus 59, 599-617.

Gritsoun, A. (2001). Fluctuation-dissipation theorem on attractors of atmospheric models. Russian Journal of Numerical Analysis and Mathematical Modelling 16(2), 115-133.

Gritsun, A. and G. Branstator (2007). Climate response using a three-dimensional operator based on the fluctuation-dissipation theorem. Journal of the atmospheric sciences 64(7), 2558-2575.

Gritsun, A., G. Branstator, and A. Majda (2008). Climate response of linear and quadratic functionals using the fluctuation-dissipation theorem. Journal of the Atmospheric Sciences 65(9), 2824-2841.

Hasselman, K. (1993). Optimal fingerprints for the detection of time-dependent climate change. Journal of Climate 6, 1957-1971.

Hasselman, K. (1997). Multi-pattern fingerprint method for detection and attribution of climate change. Climate Dynamics 13, 601-611.

Hegerl, G. C., H. von Storch, K. Hasselmann, B. D. Santer, U. Cubasch, and P. D. Jones (1996). Detecting greenhouse-gas-induced climate change with an optimal fingerprint method. Journal of Climate 6, 22812306.

Leith, C. (1975). Climate response and fluctuation dissipation. Journal of the Atmospheric Sciences 32(10), 2022-2026.

McComb, W. D. (1990). The physics of fluid turbulence. Oxford University Press.

Salmon, R. (1998). Lectures on geophysical fluid dynamics, Volume 378. Oxford University Press Oxford.

Zidikheri, M. J. and J. S. Frederiksen (2011). Inverse method for attribution of climate change. ANZIAM Journal 52, C823-C836.

Zidikheri, M. J. and J. S. Frederiksen (2013a). Computationally efficient methods for climate model inversion. ANZIAM Journal 54, C217-C232.

Zidikheri, M. J. and J. S. Frederiksen (2013b). Methods for estimating climate anomaly forcing patterns. Journal of the Atmospheric Sciences 70(8), 2655-2679. 